\title{
The Universal, Multiple, and Exclusive Experiences of After-Death Communication
}

\author{
James A. Houck, Ph.D. \\ Neumann College, Aston, PA
}

\begin{abstract}
Most of the research on after-death communications (ADCs) has been done from a qualitative approach, for example, using structured interviews (Devers and Robinson, 2002; Guggenheim and Guggenheim, 1996; LaGrand, 1997), which is an excellent way for people to begin telling their stories and comparing similar experiences with other bereft people. Yet there are other ways to measure the frequency and uniqueness of such experiences statistically, and to determine whether ADCs are random or purposeful to specific types of bereavement groups. As part of a larger research study on grief reactions and religious or spiritual coping methods in bereavement, I asked 162 bereft people to report if they have experienced specific types of $\mathrm{ADC}$, as it related to their most recent death of a loved one (Houck, 2004). A frequency analysis indicated three common themes: (1) universality - that is, ADCs cutting across lines of gender, age, religious preference, education levels, time since the death, and types of death; (2) multiplicity - that is, people typically experiencing more than one type of ADC from the same loved one on different occasions; and (3) exclusivity - that is, the ADCs being experienced without the assistance of a third party, such as a medium, spiritualist, or shaman. I discuss implications of these findings for mental health and healthcare providers.
\end{abstract}

KEY WORDS: after-death communication; bereavement; mental health.

James A. Houck, Ph.D., is Assistant Professor of Pastoral Counseling Studies at Neumann College. Reprint requests should be addressed to Dr. Houck at the Department of Pastoral Counseling Studies, Abessinio Building, Room 326, One Neumann Drive, Aston, PA 19014; e-mail: jambhouck@yahoo.com. 
Much in the same way that every human being has a thumbprint, grief is a common denominator in all societies. However, just as no two thumbprints are alike, no two people grieve in the same manner. In the context of grieving the death of a loved one, Kenneth Doka (1989) noted that there are specific losses that present a double bind: although most people who mourn the loss of a loved one are free to experience normal grief reactions, those whose loved ones carry a social stigma are not given the right, role, or capacity to grieve in the same way. He called this stigma-tainted process disenfranchised grief. This reaction by society arises from one or more of the following factors defined as disenfranchised grief: (1) the relationship to the deceased is not recognized because it was not based on traditional kin ties, such as in the case of divorce or of a gay, lesbian, or bisexual relationship; (2) the loss is not recognized or viewed by society as significant, such as in a perinatal death or the loss of a pet; (3) the survivor is perceived by society as not having the capacity to mourn, as may happen with children or the elderly; and (4) there are certain types of death, such as from suicide or autoimmune deficiency syndrome (AIDS) that may be too embarrassing or produce heightened anxiety in members of society (Doka, 1989).

In the context of both AIDS and suicide, the stigma imposed by society's discomfort may be based on a moral definition. Labeled as morally depraved and despised as social rejects, those with AIDS may be shunned, feared, and isolated by their communities. This reaction may be due to the socially unacceptable behavior that contributes to way AIDS is contracted, for example by intravenous illegal drug use, sexual promiscuity, or prostitution. What is socially unacceptable for many people regarding suicide is that, regardless of a person's feelings of hopelessness, despair, and possible mental illness, human beings do not have the right to hasten their own deaths.

Ironically, the stigma attached to AIDS-related and suicide deaths is transferred to those who grieve these losses. In other words, the griever also may be seen as somehow morally depraved and flawed because of his or her association with the deceased. Whether they had been spouses, life partners, parents, or caregivers, the survivors also may become disenfranchised through no fault of their own.

Traditionally, bereavement support is widely available for people to cope with their loss. People who have a strong locus of support are better able not only to work through the pain of the loss, but also to assimilate their grief and carry on with their lives (Nolen-Hoeksema and Larson, 1999). However, after-death communications (ADCs), in which bereaved individuals experience what they believe is actual 
spiritual contact with a deceased loved one, also have a place in helping people facilitate their losses, if survivors are given permission to tell their stories of how their loved ones continue to touch their lives. As part of a larger research study on grief reactions and religious or spiritual coping methods in bereavement (Houck, 2004), I asked bereft people to report if they have experienced specific types of $\mathrm{ADC}$, as it related to their most recent death of a loved one.

\section{Method}

\section{Participants}

Participants included people who were grieving the loss of a loved one to one of four types of death: cancer $(n=50)$, AIDS $(n=50)$, suicide $(n=50)$, and a sudden and unexpected death $(n=12)$. The relationship of the survivor to the deceased included any connection for which the bereaved sought support. In order to maintain confidentiality, I did not have direct contact with the participants, but instead used clinical case managers, nursing staff, and social workers from various hospice, suicide support groups, and human immunodeficiency virus (HIV)/ AIDS agencies to recruit volunteers for the study.

\section{Instruments}

Each volunteer was asked to complete a confidential survey packet and return it to the various agencies within one month of receiving it. The packets included the Grief Experience Questionnaire (Barrett and Scott, 1989), the Brief Religious and Spiritual Coping Scale (Pargament, Smith, Koenig, and Perez, 1998), and a demographic sheet. Participants were asked to note their relationship to the deceased, how much time had passed since the death of their loved one, if they had attended any bereavement support groups, and their religious affiliation.

The Grief Experience Questionnaire (GEQ) was designed to compare the differences in bereavement experiences of persons who have had a loved one commit suicide and persons who have had a loved one die from an accidental or natural death (Barrett and Scott, 1989). The measure consists of 55 items concerning the frequency of the following grief reactions: (1) multi-dimensional, (2) somatic reactions, (3) general grief reactions, (4) search for explanation, (5) loss of social 
support, (6) stigma, (7) guilt, (8) responsibility, (9) shame, (10) rejection, (11) self-destructive behaviors, and (12) unique reactions to suicide.

The Brief Religious and Spiritual Coping Scale (Brief RCOPE) was designed to be comprehensive and open to the negative and positive dimension of religious or spiritual coping: (1) to find meaning, (2) to gain control, (3) to gain comfort and closeness to God, (4) to gain intimacy with others and closeness to God, and (5) to achieve a life transformation (Pargament, Smith, Koenig, and Perez, 1998). The Brief RCOPE was used in this study to determine which of the coping variables each bereavement group employed.

\section{Specific ADC Criteria}

Based on categories of ADC developed by Bill Guggenheim and Judy Guggenheim (1996) and by Louis LaGrand (1997), participants were asked to answer the following question: "After the death of your loved one, was there ever a time when you sensed his/her presence?"

Those participants who answered "yes" were asked to indicate which of the following sensations applied to the after-death communication: (1) sense of the loved one being in the same room; (2) dreams of being visited by the deceased, (3) olfactory sensations, such as familiar scents, perfumes, or odors; (4) auditory sensations, such as voices, footsteps, or music; (5) visual sensations, such as seeing an outline or shape; (6) tactile sensations, such as feeling a presence through touch; (7) presence of a bird or animal that was symbolic of the deceased; and (8) a thirdparty message from an unknown person. I did not assign a numerical value to the various individual $\mathrm{ADC}$ criteria, but instead measured the frequency with which participants reported experiencing ADCs.

\section{Statistical Analysis}

All analyses were calculated using the Statistical Package for the Social Sciences (SPSS) Version 12.0.

\section{Results}

\section{Specific Grief-Related Demographics}

In looking at the four bereavement groups as a whole, women $(n=110)$ comprised 68 percent of the participants and men $(n=52)$ 32 percent. The range of ages was from 19 to 78 years, with a mean age 
of 44.7 years. Those who had lost a loved one to cancer $(n=50)$ were significantly older (mean age $=50.1$ years) than those who had lost a loved one to suicide $(n=50$; mean age $=44.2$ years), sudden death $(\mathrm{n}=12$; mean age $=42.1)$, or $\operatorname{AIDS}(\mathrm{n}=50 ;$ mean age $=39.6$ years $)(\mathrm{F}=$ $5.36 ; \mathrm{df}=3,158 ; \mathrm{p}=.0015$ ).

Income ranged from $\$ 20,000$ to $\$ 60,000$, with a mean income of $\$ 36,111$. With regard to highest educational level, 31 percent of the participants completed high school, 51 percent had attended college, and 18 percent had attended graduate school. No remarkable differences existed in comparing groups on educational level.

With regard to participants' relationship to the deceased, 24 percent were grieving the death of a parent; 21 percent had lost a spouse; 15 percent had lost a sibling; 14 percent had lost a child; 11 percent had lost a close friend; and 7 percent had lost a life-partner. Another 9 percent had lost an extended family member such as an uncle or grandparent. Time passed since their loved one's death ranged from 3 months to more than 5 years. Mean time that had elapsed was 26.6 months for those grieving a death from cancer, 25.7 months for those grieving a death from AIDS, 22.7 months for those grieving a sudden death, and 19.7 months for those grieving a suicide. Elapsed time since the loved one's death was not significantly different among those groups $(\mathrm{F}=1.10 ; \mathrm{df}=3,158)$.

Each of the participating agencies in the study provided bereaved individuals the opportunity to attend a support group, for the most part running for 6 to 10 weeks. However, a large number of the participants reported that they did not attend support group meetings following the death of their loved one $(n=127 ; 78$ percent $)$. I also asked participants about their religious affiliation in order to determine what kinds of religious and spiritual coping methods were used by survivors (Houck, 2004). In response, 22 percent were Catholic, 23 percent Methodist, 10 percent Presbyterian, 8 percent Lutheran, 7 percent the Metropolitan Community Church, 7 percent Baptist, 2 percent Episcopalian, and 6 percent reported no religious affiliation.

\section{$A D C s$}

Of the 162 participants, 122 (75 percent) reported that they had experienced at least one ADC since their loved one's death; these included 92 women ( 84 percent of the women) and 30 men (58 percent of the men). The remaining 40 participants ( 18 women and 22 men) reported that they did not experience any $\mathrm{ADCs}$ since their loved one's 
death. In order to conduct a descriptive analysis of the data, case summaries were used on the 122 reports. As a result, three patterns emerged:

Universality. I compared the different types of ADCs with demographic information, including type of death, survivor's gender, age, level of education, income, and religion; none of these variables showed significant differences. In other words, no one group experienced specific types of $\mathrm{ADC}$ versus another. In the larger study of which this was one part (Houck, 2004) self-identified bereavement groups (cancer, AIDS-related, suicide, and sudden death) not only had different grief reactions that were distinct to each type of death, but these groups were further distinguished by their different religious or spiritual coping methods. However, the different types of ADC were not associated with different types of bereavement, and therefore may be interpreted as having a universal nature.

Multiplicity. Analysis of the specific types of $\mathrm{ADC}$ indicated that survivors tended to experience more than one type of ADC from the deceased loved one at different times. For example, of the 59 participants (52 percent) who reported having experienced dreams of their loved ones visiting them, only 12 participants reported experiencing $\mathrm{ADCs}$ in dreams alone. The remaining 47 participants experienced dreams in combination with olfactory ADCs $(n=20)$, visual $A D C s(n=12)$, auditory $A D C s(n=7)$, and both olfactory and auditory $\mathrm{ADCs}(\mathrm{n}=8)$. Among those $63 \mathrm{ADC}$ experiencers who did not report $\mathrm{ADC}$ dreams, the primary $\mathrm{ADC}$ was olfactory $(\mathrm{n}=31)$, which was in combination with auditory $(n=16)$, both auditory and tactile $(n=5)$, and visual $(n=10)$. Other combinations are noted in Table 1 .

Exclusivity. Within the 122 participants who reported ADCs, 15 percent $(n=19)$ reported having experienced an ADC through thirdparty involvement, such as a medium, spiritualist, or shaman. The data reported did not include whether the survivors who received third-party ADCs were part of a group, such as audience participation or seminar format, or received their $\mathrm{ADC}$ during a private session with a medium or spiritualist. Nonetheless, every one of the 19 participants in this study reporting a third-party $\mathrm{ADC}$ also reported other types of $\mathrm{ADC}$ since their loved one's death in addition to the third-party communication: 6 reported olfactory ADCs, 4 reported both olfactory and auditory ADCs, 4 reported both auditory and visual $\mathrm{ADCs}, 2$ reported visual $\mathrm{ADCs}$, 2 reported symbolic animal presences, and 1 reported an $\mathrm{ADC}$ dream. 


\section{Table 1}

\section{Multiple Types of After-Death Communica- tions Reported (See Text for Combinations Involving Third-Party ADCs).}

\begin{tabular}{lc}
\hline $\begin{array}{l}\text { Combination of } \\
\text { ADC Types }\end{array}$ & $\begin{array}{c}\text { Number of } \\
\text { Participants } \\
\text { Reporting }\end{array}$ \\
\hline Dream ADC primary: & \\
dream ADC alone & \\
$\quad$ (no secondary ADC) & 12 \\
dream and olfactory ADC & 20 \\
dream and visual ADC & 12 \\
dream and auditory ADC & 7 \\
dream, olfactory, and & \\
$\quad$ auditory ADC & 8 \\
dream, sensed presence, and & \\
$\quad$ visual ADC & 6 \\
dream and symbolic animal ADC & 9 \\
Olfactory ADC primary: & \\
olfactory and auditory ADC \\
olfactory, auditory, and tactile ADC \\
$\begin{array}{l}\text { olfactory and visual ADC } \\
\text { olfactory and symbolic animal ADC }\end{array}$ \\
Auditory ADC primary:
\end{tabular}

From these data, one may conclude that ADC is not solely dependent on professional mediums, spiritualists, and/or shamans in order for them to occur. If anything, one may conclude that the third-party involvement of an $\mathrm{ADC}$ may operate more as an objective confirmation of what the survivor may have already experienced.

\section{Discussion and Implications for Counseling}

From the frequency analysis, it appears that after-death communication is experienced by a majority of people grieving the loss of a loved one. LaGrand (1997) asserted that one reason some people receive 
ADCs may be the role it plays in survivors' bereavement; that is, they help those survivors who are having difficulty accepting the reality of the death, accommodating the loss, and moving on with their lives without their loved one. The data also indicated that $\mathrm{ADCs}$ are universal in nature; that is, they occur in all socioeconomic and religious groups, types of death, and at various times after the death, and none of those variables were associated preferentially with any specific type of $\mathrm{ADC}$ over another. In addition, those who reported experiencing $\mathrm{ADCs}$ tended to report having experienced more than one type. In fact, the majority reported two to four types of ADC since their loved one's death. Although I did not obtain specific details of each type of $\mathrm{ADC}$, survivors indicated the various $\mathrm{ADCs}$ all were related to their most recent loved one's death. From these data, one may conclude that multiple ADCs may function as a means of confirmation or additional assurance of the deceased loved one's present state.

Finally, the majority of people reported having experienced ADCs without the assistance of a third-party contact. John Walliss (2001) suggested that the reason some people may contact a medium, spiritualist, or shaman is to maintain a sense of connection, or continuing bond, with the deceased loved one. Nonetheless, this study suggests that third-party ADCs may not occur in isolation and may also function as a means of confirmation of other experiences.

\section{Implications for Healthcare Professionals}

A common misconception about grief is that it is one-dimensional, that is, experienced solely as an emotional turmoil. Instead, grief is a multifaceted experience and may disrupt a person's physical, emotional, spiritual, social, and philosophical well-being. William Worden (2002) proposed that the grief reaction can be categorized into four areas: (1) feelings, such as sadness, anger, guilt, and anxiety; (2) physical sensations, such as tightness in the chest and throat, lack of energy, and dry mouth; (3) cognitions, such as disbelief, confusion, preoccupation, and sense of presence; and (4) behaviors, such as sleep and appetite disturbances and social withdrawal. These multifaceted experiences are considered normal reactions of grief, which usually diminish in intensity over time (Kaplan and Sadock, 1998).

\section{Meghan's Story}

Meghan was only 9 years old when she died from an AIDS-related illness. Her death was particularly troubling for all who watched her 
die, including her doctors. Meghan had contracted the AIDS virus from her mother while she was in her womb. From day one, her body had fought an uphill battle against a relentless disease, while she and her family emotionally fought against a relentless social stigma. Four months after her death, her grandfather frantically called me from his hospital bed, asking me to come that day to see him. Expecting that he had received some bad news regarding his health, I told him I would be at the hospital within the hour. When I arrived, he was sitting up in bed with a worried look on his face.

"Now, Dr. Houck," he said as I pulled up a chair close to the bed, "please don't think I'm crazy, but I need to tell you something that happened last night." He went on to tell me that he was awakened from a deep sleep around 2:00 A.M., when he saw Meghan sitting at the foot of his bed, dressed in a white nightgown. She smiled at him and waved, then vanished. He said she looked healthy, strong, and happy. He spent the rest of the night trying to make sense out of what he had experienced. I told him what he received was a precious gift, a reassurance that Meghan was safe and free from disease and pain.

Perhaps the reason Meghan came to see him was so that his last memory of her would not have been what he saw in the hospital, namely, a frail skeleton of a girl clinging to life. I also explained to her grandfather that other people have reported having ADCs and that it helped them come to terms with their losses. Through simple "permission-giving" and a little education, Meghan's grandfather was able to rise above the family stigma of AIDS that society had imposed on him for such a long time.

Understanding how ADCs may facilitate the process of mourning can be assimilated into a therapist's or mental health professional's preferred bereavement paradigm, whether that be based on stages of grief (Kübler-Ross, 1969), phases of bereavement (Parkes, 1972), or tasks of mourning (Worden, 2002). Active listening, normalizing feelings, expressing empathy, educating people on the process of mourning, and providing a safe environment where thoughts, emotions, feelings, and personal stories can be shared are crucial steps towards healing grief. Many clients may be apprehensive about sharing their $\mathrm{ADC}$ stories and feelings because they may have never been given permission to talk about the phenomenon before. Although therapists may assume this kind of permission-giving will be attractive to many, grievers may be skeptical about therapists' intentions. Too familiar with "guilt by association" judgments rendered by society, they may have difficulty accessing the therapist's empathy. 
Much of my work with the bereft is to educate them on the process of mourning. For those who have experienced an ADC, helping them explore the implications of this phenomenon can alleviate much confusion and heal the pain caused by the loss.

\section{Limitations to the Study and Future Research}

This study focused on the frequency of $\mathrm{ADCs}$ as reported by survivors from four bereavement groups: cancer, AIDS-related, suicide, and sudden death. Other types of death were not included. Therefore, a future area of research may include a comparison of ADCs experienced by survivors from multiple types of death, both across socioeconomic lines and also including comparing multiple deaths a person grieves throughout his or her lifetime.

Another limitation of this study was that it reported the frequency of ADCs from a Western cultural perspective. A future area of research would be to compare the frequency of $\mathrm{ADCs}$ from other world cultures, such as Asian, Latino, African, or Middle Eastern. In addition, personal resilience after loss (Bonanno, 2004) might also be studied to determine if any correlations exists between the type of ADC experienced and a survivor's resiliency.

Finally, this study was limited to a descriptive analysis of ADC. Perhaps a more rigorous inferential statistical design could measure grief outcomes with the existing bereavement paradigms, such as Elisabeth Kübler-Ross's stages of grief (1969), Colin Murray Parkes' phases of bereavement (1973), or William Worden's tasks of grief (2002).

\section{References}

Barrett, T. W., and Scott, T. B. (1989). Development of the Grief Experience Questionnaire. Suicide and Life-Threatening Behavior, 19, 201-215.

Bonanno, G. A. (2004). Loss, trauma, and human resilience: Have we underestimated the human capacity to thrive after extremely aversive events? American Psychologist, $59,20-28$.

Devers, E., and Robinson, K. (2002). The making of a grounded theory: After-death communication. Death Studies, 26, 241-253.

Doka, K. J. (ed.). (1989). Disenfranchised grief: Recognizing hidden sorrow. Lexington, MA: Lexington Books.

Guggenheim, B., and Guggenheim, J. (1996). Hello from heaven! A new field of research confirms that life and love are eternal. New York, NY: Bantam. 
Houck, J. A. (2004). Comparing grief reactions and religious/spiritual coping methods among cancer, AIDS-related, and suicide bereavement. Unpublished doctoral dissertation, Loyola College in Maryland, Baltimore, MD.

Kaplan, H. I., and Sadock, B. J. (1998). Kaplan and Sadock's synopsis of psychiatry: Behavioral sciences/clinical psychiatry $\left(8^{\text {th }}\right.$ ed.). Baltimore, MD: Williams and Wilkins.

Kübler-Ross, E. (1969). On death and dying. New York, NY: Macmillan.

LaGrand, L. (1997). After-death communication: Final farewells. St. Paul, MN: Llewellyn.

Nolen-Hoeksema, S., and Larson, J. (1999). Coping with loss. Mahwah, NJ: Lawrence Erlbaum.

Pargament, K. I., Smith, W., Koenig, H. G., and Perez, L. (1998). Patterns of positive and negative religious coping with major life stressors. Journal for the Scientific Study of Religion, 37, 710-725.

Parkes, C. M. (1973). Bereavement: Studies of grief in adult life. New York, NY: International Universities Press.

Walliss, J. (2001). Continuing bonds between the living and the dead within contemporary Spiritualism. Mortality, 6, 127-145.

Worden, J. W. (2002). Grief counseling and grief therapy: A handbook for the mental health practitioner $\left(3^{\text {rd }}\right.$ ed.). New York, NY: Springer. 